\title{
DIFESA EUROPEA E NUOVO ORDINE MONDIALE
}

\author{
di Domenico Moro
}

Il 2019 si è chiuso con quattro notizie relative alla politica di sicurezza di alcuni paesi: la richiesta alla Turchia, da parte di Al Serraj, di inviare truppe in Libia1; la dichiarazione del Capo di stato maggiore delle forze armate israeliane, Aviv Kochavi, secondo cui gli Stati Uniti non sono più un alleato affidabile in grado di difendere Israele dall'Iran'2; l'assegnazione ad un reggimento russo, per la prima volta al mondo, di una batteria di missili ipersonici in grado di superare qualunque barriera anti-missilistica 3 ; la diffusione, da parte della radio pubblica giapponese NHK, della notizia, poi rivelatasi falsa, del lancio di un missile nord-coreano contro il Giappone 4.

Apparentemente non vi è alcun legame tra queste notizie. In realtà, come si cercherà di dimostrare, sono collegate e, soprattutto, sollecitano

Responsabile dell'Area Sicurezza e Difesa del Centro Studi sul Federalismo.

1 Le théâtre libyen, nouvelle poudrière régionale, in "Le Monde", 28 dicembre 2019, https://www.lemonde.fr/afrique/article/2019/12/28/le-theatre-libyen-nouvelle-poudriereregionale_6024266_3212.html.

2 Top Israeli Military Leader Doubts America's Help Against Iran; Air Force Wants More $\$ \$$ For Strikes, Breaking Defense, 26 dicembre 2019, https://breakingdefense. com/2019/12/idf-bolsters-operations-vice-iran-some-question-us-support/.

3 Secondo fonti russe, la velocità che possono raggiungere i missili ipersonici installati è pari a 20 volte la velocità del suono. V. anche: Russia Says 'Avangard'Hypersonic-Missile System Now Deployed, Global security, 27 dicembre 2019, https://www. globalsecurity. org/wmd/library/news/russia/2019/russia-191227-rferl01.htm?_m=3n\%252e002a\%252e27 $66 \% 252 \mathrm{etm} 0 \mathrm{ao} 0 \mathrm{~d} 52 \mathrm{y} \% 252 \mathrm{e} 2 \mathrm{jxj}$.

4 La NHK lance par erreur une alerte au missile nord-coréen, in "Le Monde", 28 dicembre 2019, https://www.lemonde.fr/international/article/2019/12/28/la-nhk-lance-parerreur-une-alerte-au-missile-nord-coreen_6024265_3210.htm. 
un'assunzione di maggiori responsabilità europee in politica internazionale. Esse costituiscono la cornice all'interno della quale si intende sviluppare il tema oggetto di questo lavoro.

\section{I passi avanti verso una difesa europea}

A partire dalla presentazione, da parte di Federica Mogherini, della "Strategia globale per la politica estera e di sicurezza europea" a fine giugno del $2016^{5}$, con la quale, per la prima volta, viene enunciato il principio della "autonomia strategica" dell'Unione europea (UE) nel settore della difesa, a livello europeo è stata adottata una serie di decisioni che non ha precedenti nella storia del processo di unificazione europea, se non risalendo agli anni della Comunità Europea di Difesa.

In ordine di tempo, abbiamo avuto: l'istituzione del Fondo europeo per la difesa che prevede, per la prima volta da quando esiste il bilancio europeo, il finanziamento di investimenti nel settore militare; l'istituzione della "capacità di pianificazione e controllo missioni", embrione di uno Stato maggiore europeo; l'avvio della Cooperazione strutturata permanente, che ha un obiettivo di capacità militare; l'avvio del progetto di mobilità militare, per consentire alle truppe europee di spostarsi rapidamente da un paese all'altro senza impedimenti procedurali o per limiti delle infrastrutture di trasporto.

Più recentemente, a seguito dell'insediamento della Commissione europea presieduta da Ursula von der Leyen, è stata istituita una nuova direzione generale: "Industria della Difesa e Spazio". Con quest'ultima decisione e con il Fondo europeo per la difesa, la politica europea di difesa, quantomeno dal punto di vista economico, diventa una politica strutturale su cui si potranno costruire iniziative volte a rafforzarla.

\section{Una svolta strutturale nella difesa europea, benché incompiuta}

Con le decisioni appena ricordate, per quanto riguarda la difesa europea siamo di fronte ad un punto di non ritorno, che ha le sue radici in un aspetto mondiale e in un aspetto europeo.

5 F. MogherinI, Visione condivisa, azione comune: un'Europa più forte. Una strategia globale per la politica estera e di sicurezza dell'Unione europea, European Union External Action, giugno 2016. 


\subsection{L'aspetto mondiale: la svolta americana in politica estera}

Se vogliamo cogliere le grandi tendenze politiche di fondo, come la svolta in politica estera degli USA, dobbiamo accettare l'invito dello storico Fernand Braudel a prendere in considerazione l'evento che "provoca le conseguenze più importanti e numerose", in quanto "le conseguenze non si manifestano al momento, sono figlie del tempo"6.

L'aspetto mondiale della svolta europea nella politica di difesa è riconducibile al declino dell'egemonia americana sul mondo occidentale, che ha cominciato a manifestarsi all'inizio degli anni '70 del secolo scorso nel settore monetario. Si è, infatti, cominciato a parlare di moneta europea quando è diventato evidente che gli USA, con la sospensione della convertibilità del dollaro in oro (1971), non erano più in grado di assicurare l'ordine monetario mondiale. A partire da quel momento, per salvaguardare il Mercato Comune Europeo, messo in pericolo dalle oscillazioni del cambio tra le monete nazionali europee, i governi europei hanno iniziato il lungo cammino verso la moneta europea, cominciato con lo SME nel 1978 e conclusosi con la nascita dell'euro nel 1999.

Oggi si assiste all'estensione del declino americano al settore della sicurezza. Quando il Presidente Obama, nel corso del conflitto con la Libia del 2011, accusa gli europei di comportarsi da "scrocconi" ("free riders"), e afferma che 1'Alleanza Atlantica non può più essere sostenuta solo dagli USA, oppure quando il Presidente Trump ribadisce le stesse cose in termini più brutali, tutto questo equivale ad ammettere che gli USA, da soli, non sono più in grado di assicurare l'ordine politico-militare mondiale.

Le dichiarazioni ricordate all'inizio del Capo di stato maggiore israeliano, secondo il quale Israele non può più fare affidamento sugli Stati Uniti per la loro difesa dall'Iran, ne sono una conferma. Queste perplessità hanno trovato un riscontro, più recentemente, da parte di un altro dei più solidi alleati degli Stati Uniti: il Regno Unito. Ben Wallace, Ministro della difesa, in un'intervista al Sunday Times, ha ammesso che se il Regno Unito dovesse trovarsi impegnato in un conflitto, dovrà farlo senza poter contare sugli Stati Uniti, considerati ormai come un alleato su cui non si può fare affidamento ${ }^{7}$.

6 F. Braudel, Storia, misura del mondo, Bologna, il Mulino, 1998.

7 "The assumptions of 2010 that we were always going to be part of a US coalition 
Con Trump, non ci si trova di fronte ad una parentesi nella politica estera americana. Non basterà quindi sostituirlo con un altro presidente perché cambi anche la politica estera: la svolta americana in politica estera non è né democratica, né repubblicana: è semplicemente "americana". Qui di seguito, si vogliono pertanto ridimensionare le residue speranze che ci si trovi di fronte ad una politica "trumpiana".

L'Amministrazione democratica di Obama è stata, probabilmente, la prima a prendere atto che, con l'emergere della Cina, i rapporti di potere a livello mondiale erano cambiati. Infatti, la svolta più importante, nella politica estera americana è stata compiuta da Barack Obama, quando ha cambiato la strategia militare che gli Stati Uniti si erano dati a partire dalla Seconda guerra mondiale. Come sappiamo, durante il Secondo conflitto, gli USA hanno dimostrato di essere in grado di gestire contemporaneamente due guerre "ad alta intensità" (come si dice oggi) in due parti diverse del mondo: in Europa ed in Asia. Questa strategia militare è rimasta immutata fino al 2010. Con l'Amministrazione Obama, gli Stati Uniti hanno abbandonato la strategia della gestione contemporanea di due conflitti ad alta intensità, scegliendone uno, quello asiatico, relegando il territorio europeo ad area di minor importanza ${ }^{8}$.

L'Amministrazione Obama è stata anche quella che, per prima, ha dato il segnale di disimpegno dal Medio Oriente, quando ha fissato una "linea rossa" - l'uso dei gas da parte di Assad in Siria - violando la quale gli Stati Uniti sarebbero intervenuti militarmente. Tuttavia, pur a fronte di un uso accertato di gas da parte di Assad, gli Stati Uniti non sono intervenuti. Certamente, Trump gestisce la svolta in Medio

is really just not where we are going to be", in: Ben Wallace interview: We can't rely on US, Sunday Times, 12 gennaio 2020 (https://www.thetimes.co.uk/article/ben-wallaceinterview-we-cant-rely-on-us-pmwcgv398).

8 A questo ha fatto seguito l'atteggiamento di sospetto con cui l'Amministrazione Obama ha accolto l'istituzione della Asian Infrastructure Investment Bank (AIIB), decidendo di non farne parte, invitando gli alleati asiatici, come il Giappone, a fare altrettanto (V., in ordine di tempo, alcuni articoli sul punto: Obama says AIIB could be 'positive' for Asia (US president initially urged allies not to join China-led bank), in Financial Times, 28 aprile 2015 (in: https://www.ft.com/content/80271e0c-eddc-11e4-90d2-00144feab7de); China Creates a World Bank of Its Own, and the U.S. Balks in: The New York Times, 4 dicembre 2015 (https:/www.nytimes.com/2015/12/05/business/international/china-creates-an-asian-bankas-the-us-stands-aloof.html); As Allies Keep Jumping Ship, Will Obama's 'Knee-Jerk Reaction' To The AIIB End With Trump?, in: Forbes, 15 novembre 2016 (https://www. forbes.com/sites/wadeshepard/2016/11/15/as-allies-keep-jumping-ship-will-obamas-kneejerk-reaction-to-the-aiib-end-with-trump/\#70e804a51c43). 
Oriente con meno eleganza, ma la sostanza è sempre la stessa: gli Stati Uniti si stanno progressivamente disimpegnando da quell'area.

\subsection{L'aspetto europeo: insicurezza ai confini, tre trattati in scadenza}

Per quanto riguarda l'aspetto europeo di quella che si considera una svolta strutturale nel settore della difesa, ci si deve riferire ai problemi di sicurezza che riguardano in maniera specifica la nostra area. L'Unione è circondata, come mai prima d'ora, da più aree geografiche di instabilità politica e, soprattutto, militare (Est europeo, Medio Oriente, Africa settentrionale e sub-sahariana, ecc.), dalle quali, peraltro, gli Stati Uniti si stanno disimpegnando.

$\mathrm{Ma}$, soprattutto, si vuole qui attirare l'attenzione su tre trattati che sono stati firmati tra la fine degli anni ' 80 e l'inizio degli anni '90 del secolo scorso e che sono stati disdetti o che stanno per scadere: 1) il trattato sulle forze armate convenzionali in Europa (CFE): firmato nel 1988, avrebbe dovuto avere una durata illimitata, ma il 10 marzo 2015, denunciandone la violazione di fatto da parte della NATO, la Russia ha formalmente annunciato che era "completamente superato", interrompendone il rispetto; 2) il trattato firmato nel 1988 riguardante i missili a raggio intermedio (trattato INF), volto ad eliminare i missili a raggio corto (i missili con portata tra i 500 ed i $1.000 \mathrm{~km}$ ) ed intermedio (i missili di portata compresa tra i 1.000 ed i $5.500 \mathrm{~km}$ ) installati sul territorio europeo. Il 20 ottobre 2018, il Presidente Trump ha annunciato la volontà di ritirare gli Stati Uniti dal trattato, accusando la Russia di non conformità̀; 3 ) il New START (Trattato di riduzione delle armi strategiche), erede del trattato START I entrato in vigore il 5 dicembre 1994, è un trattato sulla riduzione delle armi nucleari strategiche (testate nucleari e missili balistici in grado di portare testate oltre i $5.500 \mathrm{~km}$ ). Tale accordo le limitava a 1700-2200 per ciascuna parte e proibiva l'uso di quelle multiple. È stato prorogato da USA e URSS l'8 aprile 2010 e scadrà nel 2021, ma i negoziati per estenderne la durata non sono ancora iniziati. Benché questo trattato non riguardi direttamente i paesi europei, occorre tenere presente che all'inizio degli anni ' 70 , quando la Guerra fredda toccò, probabilmente, il punto più elevato del con-

9 Il ritiro è poi stato formalizzato il 2 agosto 2019. 
fronto USA - URSS in termini di armamenti disponibili, il numero di testate nucleari americane presenti in Europa è stato stimato in oltre 7.000 , mentre oggi viene valutato in 100-150 testate ${ }^{10}$. A queste ultime, ne vanno aggiunte circa 300 dell'arsenale francese e 200 di quello inglese ${ }^{11}$.

L'idea alla base dei tre trattati era fondata su un duplice assunto: che USA e URSS avrebbero continuato ad avere lo stesso peso sulla scena mondiale che avevano avuto a partire dalla fine della Seconda guerra mondiale e che sarebbero rimaste le uniche superpotenze a livello mondiale anche negli anni a venire.

I tre trattati hanno quindi due caratteristiche comuni, che sono anche il loro punto di debolezza: 1) risalgono al periodo immediatamente successivo alla Guerra fredda e impegnano solo due potenze: gli Stati Uniti e l'Urss (poi la Russia). Si riferiscono, cioè, ad un mondo che oggi non esiste più; 2) riguardano solo l'Europa, pertanto il loro ritiro mette in discussione la sicurezza dell'Unione europea e richiede che essa prenda l'iniziativa non solo per il loro rinnovo ma anche per la loro estensione alle potenze emergenti (come la Cina, ecc.), rafforzando il ruolo delle istituzioni multilaterali. In caso contrario, gli attuali contraenti non avrebbero alcun interesse a rinnovarli.

Il fatto che i tre trattati, firmati alla fine della guerra fredda tra Stati Uniti e URSS, siano stati violati, disdetti o che stiano per scadere, ci riporta indietro di circa trent'anni e ci presenta due possibili alternative: a) la riapertura di una nuova fase di tensioni internazionali che avranno come fulcro nuovamente il territorio europeo; b) l'apertura della strada verso

${ }^{10}$ Forces aériennes européennes et mission nucléaire de l'otan, in "Defense\& Industries" no 13, giugno 2019 (https://www.frstrategie.org/sites/default/files/ documents/ publications/defense-et-industries/2019/13-2.pdf).

${ }^{11}$ NATO Nuclear Sharing and the future of Nuclear Deterrence in Europe, The Hague Centre for Strategic Studies, 31 ottobre 2018 (https://hcss.nl/news/hcss-snapshotnato-nuclear-sharing-and-future-nuclear-deterrence -europe). Per non parlare del Trattato sui missili anti-balistici (ABM), dal quale gli Stati Uniti si sono ritirati nel 2002 e che è stata una delle prime crepe nelle relazioni tra Stati Uniti e Russia: "The first crack appeared in 2002, when the United States withdrew from the Anti-Ballistic Missile (ABM) Treaty, signed three decades earlier to prevent Washington and Moscow from deploying nationwide defenses against long-range ballistic missiles. Five years later, Russia effectively suspended another landmark agreement, the 1990 Treaty on Conventional Armed Forces in Europe, and NATO followed suit", in: The Return of Doomsday, Foreign Affairs, 9 agosto 2019, https://www.foreignaffairs.com/articles/russian-federation/2019-08-06/return-doomsday.

12 A. F. KREPINEVICH, The Eroding Balance of Terror (The Decline of Deterrence), in 
un nuovo ordine mondiale, a partire dal continente europeo. L'unica possibilità di dare seguito alla seconda alternativa è nelle mani dell'UE.

\section{Innovazioni tecnologiche e politico-organizzative nel settore militare, multipolarismo e tramonto della deterrenza}

La fine del mondo bipolare, come si sostiene in un recente articolo di Foreign Affairs, mette in crisi il concetto stesso di deterrenza ${ }^{12}$. In effetti, nella misura in cui la deterrenza si basa sull'idea di mantenere un arsenale nucleare paritetico da parte di due potenze nucleari (Stati Uniti e Russia), sufficiente a scoraggiare l'avversario dal portare il primo colpo, se le potenze nucleari diventano tre, ognuna delle quali considera le altre due come potenziali avversari, l'equilibrio nucleare come fondamento della deterrenza diventa, di fatto, impossibile.

Il pericolo più insidioso, però, è un altro e riguarda non solo l'Europa, ma il mondo intero ed è lo sviluppo tecnologico nel settore degli armamenti, la sua diffusione a livello mondiale ed i cambiamenti organizzativi cui esso può dare luogo. Si possono fare quattro esempi: lo sviluppo delle armi nucleari cosiddette "tattiche", di piccola potenza e che possono essere utilizzate in operazioni di teatro; lo sviluppo di missili ipersonici in grado di trasportare testate nucleari; la guerra cibernetica e la diffusione di "notizie false" che questa consente; la militarizzazione dello spazio ${ }^{13}$.

La prima innovazione che, secondo gli esperti, porterebbe all'eliminazione della differenza tra guerra convenzionale e guerra nucleare, è quella delle cosiddette bombe atomiche tattiche, cioè testate di potenza compresa tra 10 e 100 ton. di tnt. Questa innovazione sta portando ad una preoccupante modifica organizzativa, che prevede la possibilità di porre in capo al comandante delle operazioni di teatro la responsabilità di ricorrere all'impiego di armi nucleari tattiche ${ }^{14}$. Questa scelta

"Foreign Affairs", gennaio-febbraio 2019 (https://www.foreignaffairs.com/articles/201812-11/eroding-balance-terror).

$13 \mathrm{Vi}$ è, ovviamente, l'innovazione più recente, ma anche, in prospettiva, la più inquietante, vale a dire l'estensione al settore militare dell'intelligenza artificiale, che richiede un discorso a parte.

14 Tactical Nuclear Weapons (TNW), https://www.nti.org/analysis/articles/tacticalnuclear-weapons/.

${ }^{15}$ France: Macron annonce la création d'un commandement militaire de l'espace, in 
comporterebbe il superamento del principio, fino ad ora rispettato da tutte le parti, che spetti solo al Presidente o Capo di Stato della potenza nucleare la decisione di ricorrere all'uso dell'arma nucleare. Se questa regola viene rotta e il comandante delle operazioni di teatro può decidere il ricorso all'arma nucleare, sia pure considerata "tattica", va da sé che, di fatto, si aprirebbe la via ad un'escalation incontrollabile di un eventuale conflitto locale.

La seconda innovazione, quella dei missili ipersonici, vale a dire missili in grado di trasportare testate nucleari ad una velocità che può arrivare fino a venti volte la velocità del suono, è stata resa operativa con la recente decisione della Russia, già ricordata, di dotare un reggimento con questi sistemi d'arma. L'impiego di questi missili riduce il tempo di risposta di un potenziale avversario - che è già oggi molto contenuto e pari a circa un'ora - a sei minuti, vale a dire che la risposta sarebbe praticamente impossibile. Questo significa che i contendenti sono costretti a mantenere lo stato di allarme sui livelli massimi $24 \mathrm{su}$ 24 ore e che lo spazio per eventuali trattative è nullo.

La terza innovazione riguarda la guerra cibernetica. Quest'ultima va valutata in relazione all'innovazione precedente, in quanto si tratta di uno strumento in grado di interferire con i sistemi di comando e controllo dell'avversario, in modo da mettere fuori uso i suoi sistemi di difesa e di attacco. Essa è anche lo strumento attraverso il quale si possono diffondere false notizie. L'esempio della radio pubblica giapponese NHK ricordato all'inizio, che, tra i suoi compiti, ha anche quello di monitorare e avvisare i cittadini dell'arrivo di un missile sul territorio giapponese, è emblematico. L'installazione di missili ipersonici non concederebbe più, di fatto, il tempo di verificare se la notizia del lancio di un missile nordcoreano contro il Giappone sia vera o falsa e una risposta militare immediata diventerebbe pressoché obbligata.

L'ultima innovazione, più di carattere organizzativo che tecnologica, riguarda la militarizzazione dello spazio. Nel mese di luglio dello scorso anno, la Francia ha annunciato la creazione di un "comando militare dello spazio"15. Il mese successivo, Trump ha deciso di istituire lo United States Space Command, il cui obiettivo è quello di istituire un comando unico in grado di coordinare tutte le strutture esistenti

"Le Soir", 13 luglio 2019, https://www.lesoir.be/236422/article/2019-07-13/francemacron-annonce-la-creation-dun-commandement-militaire-de-lespace.

Questa decisione, per la verità, è poco credibile. L'Ue è la proprietaria delle due 
quale sesta branca delle forze armate americane ${ }^{16}$. Sembra che anche l'Italia, come è stato dichiarato nelle settimane scorse, abbia intenzione di andare in questa direzione. In ogni caso, quello che va sottolineato è che ci si trova di fronte a decisioni che riguardano un settore al di fuori di qualunque regolamentazione mondiale e quindi soggetto ad eventi accidentali.

L'insieme di queste innovazioni sta convincendo un numero crescente di opinionisti a ritenere possibile che un conflitto possa essere scatenato per un banale errore umano o tecnico ${ }^{17}$.

\section{Verso un nuovo ordine mondiale: la "Casa comune europea"}

Come aveva fatto notare a suo tempo Michael Gorbaciov, "entrata nell'era nucleare, l'umanità ha perduto la sua immortalità"18. Questo è il senso profondo della svolta epocale che è stata compiuta con l'impiego militare dell'energia nucleare. È dunque per questo che occorre

infrastrutture spaziali decisive per la difesa e la conduzione di operazioni militari: i sistemi satellitari Galileo e Copernicus. Attraverso Ariane Espace l'Europa ha il monopolio virtuale dei vettori per il lancio di satelliti nello spazio. Non si vede, dunque, come la Francia possa, isolatamente, gestire una efficace struttura spaziale.

16 Trump Authorizes a Space Command. Next, He Wants a Space Force, jn "The New York Times", 29 agosto 2019,: https://www.nytimes.com/2019/08/29/us/politics/trumpspace-command-force.html? searchResultPosition=1. Le principali branche delle forze armate americane sono: aviazione, esercito, guardia costiera, marina e marines.

${ }^{17}$ Non vi è solo l'esempio della notizia diffusa dalla radio pubblica giapponese. A questo proposito si possono citare altri due drammatici casi che sembrano confermare i timori che un conflitto possa aver luogo a seguito di un incidente, come nel caso di conflitti locali, come successo con l'abbattimento, nei cieli dell'Ucraina, del velivolo della Malaysia Airlines, oppure a seguito di un clima di generale esaltazione, come l'abbattimento del velivolo della Ukraine International Airlines, a Teheran, in conseguenza dell'uccisione del generale iraniano Ghassem Soleimani (si veda Un rapport confirme que le vol de la Malaysia Airlines MH17 a été abattu, in "Le Monde", 9 settembre 2014: https://www.lemonde.fr/international/article/2014/09/09/un-rapport-confirme-que-le-vol-de-la-malaysia-airlines-mh17-aete-abattu_4484283_3210.html; Crash du Boeing 737 en Iran : l'avion a bien été touché par deux missiles, selon un rapport d'enquête, in "Le Monde", 21 gennaio 2020, https://www.lemonde.fr/international/article/2020/01/21/crash-du-boeing-737-en-iran-1avion-a-bien-ete-touche-par-deux-missiles-selon-un-rapport-d-enquete_6026664 3210.html.

18 G. VACCA, La sfida di Gorbaciov (Guerra e pace nell'era globale), Roma, Salerno Editrice, 2019, p. 40.

${ }^{19}$ Discours du Président de la République à la conférence des ambassadeurs, 27 
invertire la pericolosa tendenza che la scadenza dei trattati prima ricordati rischia di mettere in moto, riportando indietro di trent'anni il continente europeo $\mathrm{e}$, con il passaggio dal bipolarismo al più insicuro multipolarismo, il mondo intero.

La via da seguire, per cercare di mettere sotto controllo la crescente insicurezza, è stata indicata dalla Francia, il paese che, per ragioni storiche e per tradizione, è probabilmente il più sensibile ai mutamenti degli equilibri di potere in Europa e nel mondo. Il Presidente francese Emmanuel Macron, nel discorso agli ambasciatori francesi, alla fine del mese di agosto dello scorso anno, e nel discorso all'Assemblea parlamentare del Consiglio d'Europa, ha affermato, rispettivamente, che "il nous faut construire une nouvelle architecture de confiance et de sécurité en Europe, parce que le continent européen ne sera jamais stable, ne sera jamais en sécurité, si nous ne pacifions pas et ne clarifions pas nos relations avec la Russie"19 e che "nous avons forgé ici à l'échelle d'un continent et malgré tous les vents contraires une architecture commune au nom de la grande fraternité européenne dont Victor Hugo rêvait, avec la volonté de bâtir la maison commune européenne, évoquée par Mikhaïl Gorbatchev devant cette assemblée en 1989"20. Macron ha quindi recuperato la proposta di Gorbaciov di una "Casa comune europea" e di Mitterrand di una confederazione europea che comprenda anche la Russia.

La Casa comune europea, proposta da Gorbaciov nel luglio del 1989 e quella di Mitterrand per una confederazione europea, avanzata alla fine dello stesso anno, avevano, tra gli altri, i seguenti obiettivi: sicurezza collettiva e massimo disarmo (nucleare, chimico e convenzionale); risoluzione pacifica dei conflitti; cooperazione economica e commerciale ${ }^{21}$. Come è noto, queste proposte non sono state attuate, per responsabilità americana ma anche europea, in quella fase non an-

agosto 2019 (https://www.elysee.fr/emmanuel-macron/2019/08/27/discours-du-president-de-la-republique-a-la-conference-des-ambassadeurs-1).

${ }^{20}$ Discours d'Emmanuel Macron au Conseil de l'Europe à Strasbourg, $1^{\circ}$ ottobre 2019 (https://www.elysee.fr/front/pdf/elysee-module-14400-fr.pdf).

${ }^{21}$ L'idea di una "difesa difensiva" e di "sufficienza militare" aveva i seguenti obiettivi: a) " $i$ due concetti presuppongono una struttura delle forze armate di uno Stato, in modo tale che siano sufficienti a rispondere a una possibile aggressione, ma insufficienti a compiere azioni offensive"; b) "il primo passo verso questo obiettivo è il ritiro controllato dalle aree di confine delle armi nucleari e di altre armi offensive". L'idea di Gorbaciov e di Mitterrand era quella di dar vita ad un'associazione che comprendesse UE e URSS e che avesse come obiettivo la sicurezza europea, organizzata attorno all'idea di una "di- 
cora dotata - come non lo è tuttora - di una politica estera estesa alla sicurezza ed alla difesa ${ }^{22}$.

Con la fine dei tre trattati precedentemente citati, se si vuole evitare una nuova escalation militare in Europa - ma anche evitare una sorta di "balcanizzazione" del continente, di fronte al ritiro americano e al riarmo russo - occorre riprendere quelle proposte, sia pure collocandole nella prospettiva di una loro graduale realizzazione.

Macron ha infatti intuito quella che può essere la strada da seguire per una politica costruttiva nel settore della sicurezza con lo Stato russo, ma occorrerà allargare il quadro di riferimento, consapevoli però che ci si muove su un terreno ancora largamente incerto e dove occorreranno fantasia e riflessioni approfondite al fine di trovare soluzioni percorribili, anche se difficili. Qui di seguito, si avanzano solo alcune idee che tengono conto anche della crisi che sta attraversando l'Alleanza atlantica ed il suo braccio militare, la NATO.

Dal punto di vista istituzionale, non si parte da zero e, ancora una volta, il Presidente Macron ha indicato la direzione che si può seguire. Come già accennato prima, intervenendo nel corso della cerimonia per il $70^{\circ}$ anniversario della fondazione del Consiglio d'Europa (CDE), ha individuato in quest'ultimo l'istituzione attraverso la quale si può con-

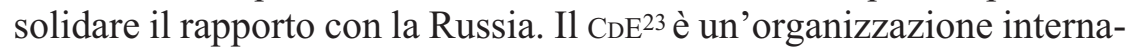
zionale, istituita nel 1949, il cui obiettivo è quello di difendere i diritti umani, la democrazia e lo stato di diritto in Europa ed è dotata di un'Assemblea parlamentare di cui fa parte anche la Russia, mentre Stati Uniti e Canada ne fanno parte come osservatori. Esso può dunque costituire il punto di partenza per cominciare ad impostare un rapporto cooperativo con la Russia. Tuttavia, non è uno strumento sufficiente, perché tra i suoi scopi non vi è la politica di sicurezza.

fesa difensiva" ("una struttura delle forze armate di uno Stato, in modo tale che siano sufficienti a rispondere a una possibile aggressione, ma insufficienti a compiere azioni offensive").

22 Gli Stati Uniti si opposero ad un finanziamento del FMI di 14 miliardi di dollari chiesto da Gorbaciov, mentre tre anni dopo sostennero un finanziamento al Messico di circa 50 miliardi di dollari per consentirgli di superare la sua crisi finanziaria (si veda M. CAmdessus, Drawing Lessons from the Mexican Crisis: Preventing and Resolving Financial Crises - the Role of the IMF, 22 maggio 1995, https://www.imf.org/en/News/Articles/2015/09/28/04/53/spmds9508).

${ }^{23}$ Da non confondere con il Consiglio europeo o il Consiglio dei ministri dell'UE.

${ }^{24}$ Come è noto, essi hanno dato vita al North American Free Trade Agreement 
Occorrerà pertanto attivare un altro organismo, l'Organizzazione per la sicurezza e la cooperazione in Europa (OSCE) che, tra le sue finalità, ha proprio la politica di sicurezza sull'intero continente eurasiatico e di cui fanno parte, oltre ai paesi membri del CDE, gli Stati Uniti, il Canada e le repubbliche euro-asiatiche. L'osce, però, ha il limite di essere un organismo sui generis, in quanto gli Stati Uniti si sono sempre rifiutati di farne oggetto di un vero e proprio trattato.

Per rafforzarne il ruolo sarà quindi necessario consolidarla con un trattato, al fine di dotarla di un minimo di formali istituzioni comuni: poiché i compiti dell'OSCE e del CDE in parte si sovrappongono ed in parte sono complementari, la via che si potrebbe percorrere potrebbe essere quella di unificarli, al fine di rendere anche l'OSCE l'oggetto di un trattato. Non si insisterà mai abbastanza su quest'ultimo punto, importante soprattutto per il rapporto con gli Stati Uniti. Questi ultimi non hanno mai fatto l'esperienza di condividere con altri paesi delle istituzioni comuni come, ad esempio, un' assemblea parlamentare sovranazionale 24.

Questa politica di coinvolgimento degli UsA non solo va perseguita e rafforzata: al fine di consolidare la sicurezza del continente, occorrerà anche lavorare affinché l'Alleanza Atlantica diventi, in prospettiva, parte dell'istituzione che nascerà dall'accorpamento tra $\mathrm{OSCE}$ e $\mathrm{CDE}^{25}$. Nel frattempo, occorrerà approfondire attraverso quale politica la nuova istituzione potrebbe avviare, sia pure gradualmente, un rapporto di collaborazione che sia nell'interesse dell'UE e della Russia ${ }^{26}$.

(NAFTA) con Canada e Messico, ma questa associazione non è mai stata accompagnata dalla creazione di istituzioni comuni ai paesi coinvolti. Si potrebbe obiettare che la NATO è dotata di un'assemblea parlamentare, così come l'OSCE, ma nel primo caso essa ha un ruolo poco più che simbolico, in quanto il trattato istitutivo dell'Alleanza Atlantica non la prevede per nulla, mentre nel secondo caso l'OSCE non è ancora oggetto di un trattato e quindi la sua assemblea è, forse, meno che simbolica.

${ }^{25}$ Per quanto riguarda la struttura militare dell'Alleanza Atlantica, vale a dire la NATO, i tempi del coinvolgimento della Russia saranno verosimilmente più lunghi.

${ }^{26}$ Poiché la nuova istituzione che nascerebbe dall'accorpamento di OSCE e CDE avrebbe tra i suoi compiti più importanti quello della sicurezza euro-atlantica, occorrerà porsi il problema dei rapporti tra questa istituzione e l'Alleanza Atlantica, cui si qui vuole solo accennare. Anche qui, è bene ricordare che non si parte da zero. Infatti, a partire dalla seconda metà degli anni '90 del secolo scorso, è stata promossa una serie di accordi tra Alleanza Atlantica e Russia. Nel 1994, la Russia è entrata a far parte del Partenariato per la Pace (PPP), di cui fanno parti gli Stati aderenti alla NATO e le ex-repubbliche sovietiche. Nel 1997, la relazione NATO-Russia ha fatto un salto di qualità con l'approvazione dell'Atto istitutivo 
Ancora una volta, è il discorso di Macron agli ambasciatori francesi ad indicare la strada, quando ha osservato che il sistema politico-economico russo è debole ("cette grande puissance qui investit beaucoup sur son armement, qui nous fait si peur a le produit intérieur brut de l'Espagne, a une démographie déclinante et un pays vieillissant, et une tension politique croissante"). Questa osservazione sembra spiegare il senso dell'intervento dell'Ambasciatore russo a Bruxelles, Vladimir Chizhov, il quale, nel corso di un convegno sulla politica estera, organizzato dalla Körber Stiftung di Berlino, nel novembre del 2018, ha proposto l'istituzione di una "zona economica" tra la UE e l'Unione economica euro-asiatica ${ }^{27}$. Questa prospettiva è stata rilanciata, recentemente, dal ministro degli esteri russo, Sergej Lavrov, nel corso di un'intervista ad un quotidiano italiano 28.

I contenuti di questa zona economica che si dovrebbe realizzare, non sono stati precisati, ma la proposta dovrebbe essere presa in seria considerazione. Va da sé che i progressi in questo campo dovranno svilupparsi tenendo conto dell'avvertimento di Macron ("avancer sur cette voie, encore une fois sans naïveté"), e quindi progredire solo in parallelo

sulle relazioni reciproche, cooperazione e sicurezza (Nato-Russia Founding Act on Mutual Relations, Cooperation and Security) (si veda R. AlCARO, V. BRIANI, Le relazioni della Russia con la Nato e l'Unione europea, Istituto Affari Internazionali, novembre 2008). Inoltre, nello stesso anno, i membri della PpP sono entrati a far parte del Partenariato Euro-Atlantico, che ha un ruolo di consultazione e coordinamento. Nel 2002, in occasione del vertice NATO di Pratica di Mare, i Capi di Stato e di Governo dei Paesi alleati e della Russia hanno firmato la Dichiarazione "Relazioni NATO-Russia: una nuova qualità", dalla quale è scaturita la creazione di una nuova ed originale formula di partenariato: il Consiglio NATO-Russia, ad oggi il principale strumento di collegamento tra paesi NATO e Russia. (si veda M. MORENO, La cooperazione Nato-Russia, https://www.esteri.it/mae/doc/1503.pdf). I fatti della Georgia (2008), l'annessione illegale della Crimea (2014) e la guerra civile nel nord-est dell'Ucraina (2014) hanno indubbiamente indebolito questa rete di rapporti tra paesi occidentali e Russia. Ma la posizione di apertura verso quest'ultima sul tema della sicurezza, rilanciata da Macron nell'agosto del 2019 e ribadita recentemente durante il vertice franco-polacco di inizio febbraio (si veda Déclaration conjointe du Président Emmanuel Macron et de M. Andrzej Duda, Président de la République de Pologne, https://www.elysee.fr/emmanuel-macron/2020/02/03/declaration-conjointe-du-president-emmanuel-macron-et-de-m-andrzejduda-president-de-la-republique-de-pologne), sono il segnale di un'evidente preoccupazione per il futuro della sicurezza del continente europeo.

27 https://club.bruxelles2.eu/wp-content/uploads/2018/11/nouvelleeresecuritechizhovberlin@rus181127.pdf.

${ }^{28}$ Lavrov teme un ruolo Nato in Libia: missioni militari sotto ombrello Onu, in "La Stampa", 19 febbraio 2020.

${ }^{29}$ Come è stato fatto notare "Le espressioni "NATO" e "Alleanza Atlantica" o più sem- 
ad avanzamenti nel settore della sicurezza. Resterebbe solo da sottolineare che la riconciliazione euro-russa equivarrebbe, per il continente euro-asiatico, a quello che la riconciliazione franco-tedesca ha rappresentato per l'Europa: la pacificazione dell'intero continente euro-asiatico e quindi un passo avanti verso forme crescenti di integrazione.

Il punto cruciale, però, è ancora un altro, in quanto l'obiettivo di una sicurezza continentale stabile non può fondarsi su trattati o alleanze di tipo tradizionale, ma occorrerà trovare soluzioni innovative.

\section{Altiero Spinelli e il superamento delle organizzazioni internazionali}

Il problema è che quando si parla di $\mathrm{CDE}$ e, a maggior ragione, dell'OSCE, anche nel caso in cui si dovesse arrivare ad un accorpamento delle due organizzazioni, si tratterebbe pur sempre di organizzazioni internazionali classiche, il cui processo decisionale sarebbe condizionato dal voto unanime o per consenso degli Stati partecipanti. Occorre dunque vedere se, ed a quali condizioni, questo tipo di organizzazioni può evolvere verso forme più avanzate di integrazione. Le considerazioni sviluppate a suo tempo da Spinelli a proposito dell'Alleanza Atlantica, forniscono degli spunti utili anche per il CDE e l'OSCE.

\subsection{Spinelli e l'Alleanza Atlantica: verso alleanze di tipo nuovo}

Spinelli, riflettendo sulla natura delle organizzazioni internazionali nel contesto di un articolo dedicato alla NATO, aveva colto un importante elemento di novità che differenziava l'Alleanza Atlantica dalle alleanze tradizionali29. Egli aveva individuato non soltanto l'originalità di questa alleanza, ma soprattutto le condizioni per il superamento delle organiz-

plicemente "Alleanza" vengono correntemente utilizzate come sinonimi negli ambienti degli addetti ai lavori [...] sebbene le espressioni citate abbiano significati differenti. La prima fa riferimento ad una connotazione giuridica che comprende sia il Patto che la struttura permanente. La seconda, invece, ha un significato più politico indicando il Patto che lega gli Stati interessati al Trattato" (si veda Stato Maggiore dell'Aeronautica, La NATO . Cenni sulle strutture organizzative e sui principali aspetti procedurali, 2013; in:

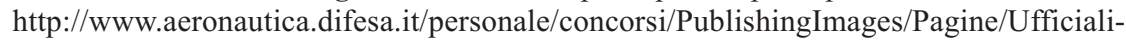
Ruolo-Speciale/Sinossi\%20NATO\%202013-2014.pdf). La medesima distinzione viene fatta nel presente lavoro, in quanto l'Alleanza Atlantica è nata nel 1949 e la sua struttura mi- 
zazioni internazionali in generale, in modo da consentire loro di evolvere in vere e proprie istituzioni sovranazionali tendenzialmente democratiche. Spinelli, infatti, aveva cominciato con il far notare che "formalmente il Patto Atlantico è un'alleanza difensiva fra Stati sovrani, ma esso è in realtà fondamentalmente diverso dalle tradizionali alleanze che si incontrano nella storia europea. Queste, infatti, finché il comune nemico non avesse commesso una effettiva aggressione, restavano, per così dire, latenti, lasciando che ciascuno degli alleati portasse avanti la propria politica estera e militare senza avere alcuno specifico impegno verso gli altri. La lettera del Patto Atlantico è conforme a questa concezione, ma la realtà è andata rapidamente oltre. [...] il pericolo era tale e di tali dimensioni da imporre una difesa militare permanente e relativamente unificata".

Nelle sue osservazioni, Spinelli è andato anche oltre, quando ha affermato che "poiché quest'organizzazione [1'Alleanza Atlantica] è istituzionalizzata, quel che abbiamo innanzi non è, in realtà, un'alleanza classica, ma una vera e propria confederazione militare". Ma, continuava, "come tutte le altre confederazioni che hanno significato qualcosa nella storia, anche questa è vitale solo perché e finché i suoi membri non sono tutti uguali, ma ce n'è fra loro uno più 'eguale degli altri', una potenza egemonica" 30 . Se la presenza di una potenza egemonica costituiva la condizione per il suo funzionamento, essa era però anche il suo limite. In effetti, Spinelli aveva individuato due generi di quelli che ha chiamato i 'centri di azione comune'. "Il primo

litare è stata istituita nel 1951, con Dwight D. Eisenhower come primo comandante in capo. Con il tempo, il termine NATO ha finito per essere associato unicamente alla struttura militare di un'alleanza che, inizialmente, voleva essere una tradizionale alleanza politico-militare. A ciò ha contribuito il fatto, unico al mondo e rilevato da Spinelli, che la struttura militare è diventata permanente (per le origini della NATO e della sua struttura militare, si veda L. S. KAPLAN, The United States and NATO. The formative years, Lexington, University Press of Kentucky, 1984). A supporto della necessità di operare questa distinzione sta il fatto che, come si ricorderà, la Francia di De Gaulle, nel marzo del 1966, uscì dal comando integrato della NATO, ma non dall'Alleanza Atlantica ("L'alliance atlantique se poursuivra pour ce qui concerne la France" mais aucune réforme de l'O.T.A.N. "ne pouvant être envisagée" la France entend rétablir sur son territoire sa pleine souveraineté, in "Le Monde", 10 marzo 1966, https://www.lemonde.fr/archives/article/1966/03/10/1-alliance-atlantique-se-poursuivra-pour-ce-qui-concerne-la-france-mais-aucune-reforme-de-1-o-t-a-n-ne-pouvant-etre-envisagee-la-france-entend-retablir-sur-son-territoire-sa-plein_2700392_1819218.html). Ancora oggi, dopo essere rientrata nella NATO nel 2009, la Francia non fa parte dell'unità di pianificazione nucleare di quest'ultima.

30 A. SPINELli, Coordinamento e integrazione nella NATO, in A. SPINELLI, “ L'Europa tra Ovest ed Est”, Bologna, il Mulino, 1990, pp. 156-157. 
genere - osservava Spinelli - è costituito dalla presenza di uno stato talmente predominante su tutti gli altri da riuscire ad imporre senza troppe difficoltà il suo punto di vista e le sue decisioni; il secondo genere è costituito da strutture alle quali tutti gli stati membri riconoscono una certa autonomia in certi determinati campi, e che sono tenuti a svolgere questa loro azione a nome e nell'interesse di tutta l'organizzazione".

Il primo genere individuato da Spinelli corrisponde all'esperienza dell'Alleanza Atlantica, dalla sua nascita fino ad oggi, in quanto nessuno, nemmeno Macron, che pur si è dimostrato il più critico nei confronti di questa organizzazione ${ }^{31}$, ne mette in discussione il ruolo nella difesa europea ${ }^{32}$. Il fatto è che l'Alleanza Atlantica non è riuscita a fare il passaggio dal "centro di azione comune" del primo genere al secondo, nonostante il Segretario generale della NATO e il comandante in capo delle forze armate di questa organizzazione siano responsabili di fronte all'Alleanza in quanto tale - e non di fronte a singoli governi nazionali -, vale a dire la condizione che Spinelli vedeva come necessaria per rafforzarne l'inclinazione sovranazionale. A posteriori, si deve concludere che il peso della potenza egemone all'interno dell'Alleanza Atlantica, gli USA, si è dimostrato troppo forte e quindi un ostacolo insuperabile per ulteriori sviluppi in senso sovranazionale ${ }^{33}$.

Se l'ostacolo ad uno sviluppo più schiettamente sovranazionale è stato il rapporto squilibrato tra gli Stati Uniti da una parte e tutti gli altri Stati membri dell'Alleanza Atlantica dall'altra, esso potrebbe essere superato solo con la presenza all'interno di questa organizzazione di una comunità politica di peso paragonabile a quella americana e che favorisca uno sviluppo in senso più sovranazionale dell'organizzazione. Questa comunità politica, pur nella sua incompletezza, non può che essere l'Unione europea.

${ }^{31}$ Emmanuel Macron warns Europe: NATO is becoming brain-dead, in " The Economist", 7 novembre 2019.

32 "Je l'ai dit, l'Alliance atlantique est et restera un socle indispensable de notre sécurité", dal discorso pronunciato da Macron a Varsavia il 3 febbraio 2020; "L'OTAN et l'Europe de la défense sont les deux piliers de la sécurité collective européenne", dal discorso pronunciato da Macron all'École de Guerre francese l'8 febbraio 2020.

33 Il documento di Spinelli fa pensare che egli ritenesse possibile uno sviluppo della NATO sul modello della Comunità europea, dove la prima avrebbe avuto una vera e propria competenza, indipendente dagli Stati membri, nel settore militare e la seconda una competenza economica. 


\subsection{Spinelli e la 'scintilla di sovranazionalità'}

Spinelli, in effetti, ha fatto un'ulteriore osservazione. Le organizzazioni internazionali possono avere un carattere evolutivo, purché soddisfino ad alcune condizioni. "Questo processo di creazione di organizzazioni internazionali contenenti almeno una scintilla di vita sovranazionale - aveva osservato Spinelli - può svilupparsi solo se è concepito nella prospettiva della creazione di un ordine federale non regionale, ma mondiale, del quale queste organizzazioni parziali siano solo parti o preludi. Si può sorridere dinanzi a questa prospettiva, ma al di fuori di essa tutte le parziali organizzazioni internazionali diventano futili e non resta che la prospettiva classica della rivalità di tutti gli Stati contro tutti" 34 . Spinelli aveva quindi individuato due condizioni: la prima era che le organizzazioni internazionali dovevano contenere "almeno una scintilla di vita sovranazionale"; ma era soprattutto la seconda condizione ad essere decisiva, vale a dire che esse dovevano essere concepite "nella prospettiva della creazione di un ordine federale non regionale, ma mondiale". Si tratta dunque di vedere se, ed in che misura, CDE e OSCE le soddisfano entrambe.

Il primo punto su cui dobbiamo quindi interrogarci è la "scintilla di vita sovranazionale" che le può far progredire. Per quanto il problema sia aperto ad una riflessione che è ancora ai suoi inizi, possiamo dire che non è sufficiente, come invece sosteneva Spinelli, che i loro responsabili siano persone scelte dall'istituzione in quanto tale e che quindi - in virtù di questa legittimazione derivante dall'insieme e non da una parte - si sentano libere da condizionamenti nazionali e portate a fare l'interesse dell'istituzione sovranazionale. Occorre che questa politica sia sostenuta da un potere che, a sua volta, per inclinazione, se non per interesse, sostenga il rafforzamento dell'istituzione sovranazionale. Questo potere non può che essere l'Unione europea in quanto, per la sua storia e per la dichiarata e fattiva propensione a sostenere lo sviluppo delle istituzioni multilaterali, può costituire la "scintilla di vita sovranazionale" di cui parla Spinelli.

Si è già osservato che gli Stati Uniti non hanno mai fatto- o non hanno mai voluto fare - l'esperienza di condividere con altri paesi istituzioni parlamentari comuni. I paesi che, invece, hanno dato vita all'UE

${ }^{34}$ A. Spinelli, Coordinamento e integrazione nella NATO, cit., pp. 261-262. 
e ne hanno accettato il progressivo rafforzamento, hanno anche fatto l'esperienza, unica nel mondo moderno, di condividere istituzioni democratiche comuni e questa attitudine si è trasferita all'interno stesso delle istituzioni europee. Così, l'UE con la politica dell'allargamento ha condiviso con un numero crescente di paesi le istituzioni democratiche europee. In particolare, l'allargamento ad Est ha dimostrato che una transizione pacifica allo stato di diritto e ad un'economia di mercato regolata è possibile ed in tempi ragionevoli, sia pure con residue difficoltà. Con la politica di aiuti allo sviluppo, l'UE ha dato vita ad istituzioni parlamentari comuni con i paesi destinatari degli aiuti, come l'Assemblea parlamentare paritetica con i paesi ACP (Africa, Caraibi e Pacifico) e l'Assemblea parlamentare euro-mediterranea che riunisce, oltre ai paesi UE, quindici paesi del Nord Africa, del Medio Oriente e del Sud Europa. Essa, infine, promuove attivamente i processi di integrazione regionale.

Resta da esaminare la seconda condizione messa in luce da Spinelli, vale a dire se la nuova istituzione per la sicurezza si porrebbe o meno in una prospettiva mondiale. Questa condizione verrebbe soddisfatta solo se il CDE e l'OSCE diventassero un'unica istituzione che comprendesse quindi, oltre alla Russia, anche il Nord America e i paesi eurasiatici. La nuova istituzione sarebbe una comunità di sicurezza intercontinentale che dovrà però non solo essere aperta alla partecipazione di altri paesi che vorranno condividere con essa le politiche nel settore della sicurezza, ma essere al servizio delle Nazioni Unite.

L'UE può quindi ridare un ruolo al CDE ed all'OSCE e, in prospettiva, porre il problema del loro accorpamento, ma dovrà compiere alcuni passi indispensabili, quali: l'ingresso dell' $U$ E in quanto tale in entrambe le organizzazioni, così come nell'Alleanza Atlantica, accanto, in una fase transitoria, ai rappresentanti nazionali; il passaggio al voto a maggioranza qualificata in politica estera, come previsto dal programma della Presidente della Commissione europea, Ursula von der Leyen ${ }^{35}$; l'avvio di una difesa europea autonoma minima, mettendo ad esempio a disposizione dell'UE, in modo permanente, risorse e mezzi militari attualmente impegnati nelle 16 missioni al di fuori dei confini dell'UE.

35 In questo contesto si può ricordare la proposta della Commissione europea di passare al voto a maggioranza qualificata, sfruttando la clausola passerella, in tre campi: 1) rispondere collettivamente alle violazioni dei diritti umani; 2) applicare sanzioni efficaci; 3) avviare e gestire le missioni civili di sicurezza e di difesa. 


\section{La difesa europea e gli ostacoli sul suo cammino}

La difesa europea si farà se il modello di riferimento non sarà lo Stato nazionale di stampo europeo, ma se si guarderà all'esperienza delle unioni federali, in particolare a quella americana. Quest'ultima, fino a che si sono mantenute vitali le istituzioni federali sulla base delle quali era nata, aveva una struttura militare che il costituzionalista australiano Kenneth Wheare qualificò come dual army, in quanto fondata sulle prevalenti milizie statali e su un piccolo esercito federale. L'Europa farà passi avanti verso la difesa se si darà un modello simile, ma con una precisazione ${ }^{36}$.

Questo modello di difesa corrisponde, in termini istituzionali più solidi, all'idea della "difesa difensiva" di cui parlava Gorbaciov, e costituisce anche un buon punto di riferimento in base al quale impostare, senza 'ingenuità', come dice Macron, una politica di sicurezza continentale con la Russia. Se quest'ultima, in prospettiva, non accogliesse un modello simile a quello della dual army, sarebbe difficile per l'Europa adottarlo e mantenerlo in permanenza. Nel giro di poco tempo, finirebbe per abbracciare un modello di difesa tradizionale.

In ogni caso, per avanzare verso una difesa europea, ad oggi ci sono delle difficoltà politiche di cui occorre tenere conto e che si possono precisare ricordando quanto scrisse Tommaso Padoa-Schioppa a proposito della natura della leadership ${ }^{37}$. Interrogandosi su quest'ultima, la risposta che Padoa-Schioppa ha dato è che la leadership consiste più nel dare che nel chiedere e questo, ad oggi, illumina la differenza di statura politica tra Helmut Kohl, che ha saputo abbandonare il marco per consentire la nascita dell'euro, ed Emmanuel Macron che, invece, si limita a rivendicare con insistenza un bilancio dell' eurozona, mentre non si dimostra ancora in grado di dire a che cosa la Francia è disposta a rinunciare pur di consentire di far fare passi avanti al progetto europeo.

La Francia ha tradito quattro volte il progetto europeo: nel 1954, quando l'Assemblea nazionale francese boccia il trattato istitutivo della CED; nel 1984, quando Mitterrand di fronte al Parlamento europeo si impegna a difendere il Trattato sull'Unione europea di Spinelli e poi non lo fa; nel 1991, quando Kohl si dichiara disponibile a compiere passi avanti verso l'unione politica assieme all'unione monetaria, e

\footnotetext{
${ }^{36}$ Questa prospettiva è commentata più ampiamente in: D. Moro, Verso la difesa europea. L'Europa e il nuovo ordine mondiale, Bologna, il Mulino, 2018.

37 T. PAdOA-SchiopPa, La veduta corta, Bologna, il Mulino, 2009.
} 
Mitterrand fa orecchie da mercante 38 ; nel 2005, quando i francesi bocciano il Trattato istitutivo di una Costituzione europea.

Sono passati più di tre anni, da quando Macron ha fatto il suo bellissimo discorso alla Sorbona "Pour une Europe souveraine, unie, démocratique" 39 , ma siamo ancora in attesa che ci chiarisca in che cosa consiste un'Europa sovrana, unita e democratica e, soprattutto, che ci presenti, come prevedono i trattati esistenti, un progetto per la revisione di quelli attuali, là dove la Francia ritiene che debbano essere modificati.

Credo che, con riferimento al tema di questo lavoro, non occorra molta fantasia per individuare il campo in cui l'Europa può fare il passo decisivo verso la propria unificazione e dove, quindi, ci si attende che la Francia abbia il coraggio di compiere la stessa scelta, sul terreno militare, che la Germania di Kohl ha saputo fare sul terreno monetario: la disponibilità della Francia a rinunciare ad almeno una parte delle proprie forze armate, mettendole a disposizione, in permanenza, dello Stato maggiore europeo. Senza questo passo, la Francia di Macron avrà tradito il progetto europeo per la quinta volta.

Certamente, il Presidente francese ha avuto l'idea, fatta propria dalla Presidente della Commissione europea, Ursula von der Leyen, di convocare una Conferenza sul futuro dell'Europa. Indubbiamente, si tratta di un'occasione importante che non andrà persa solo se i cittadini europei sapranno imporre ai suoi lavori un'agenda precisa. Un'agenda che comprenda l'attribuzione in capo all'Unione europea di forze armate minime in grado di eseguire missioni al di fuori dei suoi confini.

Uno stimolo importante in questa direzione potrebbe venire dall'Italia, se chiedesse che le attuali risorse europee impegnate in sedici missioni civili-militari in varie parti del mondo vengano messe a disposizione dell'UE e se l'Italia si dichiarasse disponibile a mettere a disposizione dell'UE, in permanenza, una parte delle sue forze armate, a condizione che gli altri Stati europei disponibili facciano lo stesso. In questo caso, per la Conferenza sul futuro dell'Europa, sarebbe difficile ignorare la svolta che il mondo si aspetta dall'Europa per un nuovo ordine mondiale.

${ }^{38}$ Per una più che buona ricostruzione delle vicende di quegli anni, si veda M. J. BAun, The Maastricht Treaty as High Politics: Germany, France, and European Integration, in "Political Science Quarterly", n. 4, 1995-96, pp. 605-624.

${ }^{39}$ Initiative pour l'Europe. Discours d'Emmanuel Macron pour une Europe souveraine, unie, démocratique, 26 settembre 2017: https://www.elysee.fr/emmanuel-macron/2017/09/26/initiative-pour-1-europe-discours-d-emmanuel-macron-pour-une-europe-souveraine-unie-democratique. 


\section{Osservazioni conclusive}

Il multilateralismo americano, maturato alla fine del Secondo conflitto mondiale in un contesto in cui le condizioni politiche apparivano insormontabili, ha toccato il suo apice nei primissimi anni seguiti alla fine della guerra. Dopo di allora, anche per il colpevole ritardo con cui l'Europa ha fatto passi avanti verso la propria unificazione, ha prevalso la logica della politica di potenza e il ruolo americano ha progressivamente assunto le vesti dell'egemonia, erodendo, con il tempo, l'aspetto più innovativo e nobile di quella politica. Quest'involuzione non toglie però nulla al significato rivoluzionario di quella svolta. Infatti, con essa, per la prima volta, la prospettiva della progressiva unificazione mondiale non restava più confinata nell' ambito delle aspirazioni ideali, ma diventava l'oggetto di una politica e, quindi, possibile. L'Unione Europea è la sola comunità politica, per quanto ancora incompleta, che oggi può assumersi la responsabilità di riprendere quella politica e quel cammino che sembra essersi interrotto. Solo così, e con il coinvolgimento degli Stati Uniti, il federalismo dei Padri fondatori della federazione americana, il cui significato profondo è quello di essere il modello istituzionale in grado di imbrigliare la politica di potenza, ritroverà nuovo slancio.

\footnotetext{
Abstract - Over the past three years, the European Union has adopted a number of decisions in the European defense sector. They are the most important decisions sixty years after the failure of the European Defense Community and this means that we are facing a structural change in this field. The European Commission's decision to establish the new Defense and Space Directorate-General confirms that defense policy is by now an EU structural policy.

The change of the American military strategy, with the shift of attention from European to Asian theater, as well as the progressive American disengagement from the Middle East and the questioning of the role of NATO, are all signs of a progressive weakening of the role of the USA in the world and explain European defense policy activism. The coming to an end of
}

three treaties for the reduction of conventional and nuclear weapons on European territory, are also contributing to this change of European attitude toward defence matter.

This paper analyzes a possible way out of a new arms race on the European continent, taking up the proposals of Gorbachev and Mitterrand for a "Common European home" which, by strengthening the role of the Council of Europe and the OSCE, include Russia and the United States itself. It also resumes the indications of Altiero Spinelli concerning the conditions necessary for the setting up of institutions that overcome traditional alliances in order to restore lasting peace, not only on the Eurasian continent, but also in the relations between the latter and the American continent. 